\title{
Monitoramento de aves limícolas na Salina Diamante Branco, Galinhos, Rio Grande do Norte, Brasil
}

\author{
Maria Eduarda de Larrazábal ${ }^{1}$ \\ Severino Mendes de Azevedo Júnior ${ }^{1,2}$ \\ Oldair Pena ${ }^{3}$
}

\begin{abstract}
Monitoring of the shorebirds in Diamante Branco saline, Galinhos, Rio Grande do Norte, Brazil. Monitoring of the shorebirds of the Diamante Branco saline located at the municipality of Galinhos, in the coast of the State of Rio Grande do Norte, was initiated in 1998, with qualitative surveys, bird traps using mist net, captured and banding and field observations on the diet of some species. Parameters such as constancy were verified, specific diversity was calculated through Pearson correlation. We registered 21 species of shorebirds, as follows: Pluvialis squatarola (Linnaeus, 1758), Charadrius semipalmatus Bonaparte 1825, Arenaria interpres (Linnaeus, 1758), Tring a flavipes (Gmelin, 1789), T. melanoleuca (Gmelin, 1789) and Actitis macularia (Linnaeus, 1766). It was observed the reproduction of Charadrius collaris Vieillot, 1818, C. wilsonia Ord, 1814 and Himantopus himantopus (Müller, 1776), in the surroundings of the saline evaporation tanks. The observation of Calidris himantopus (Bonaparte, 1826) in the evaporation tanks comprised the first record of the species in Northeastern Brazil. It was verified that A. interpres, $T$. flavipes, $T$. melanoleuca and Calidris pusilla (Linnaeus, 1766) feed upon Artemia franciscana (Kellog, 1996) in the first hours of the day in the evaporation tanks. The mean diversity comprised 2 and 3 , and the results of equibility showed that the species are well distributed in the samples. A positive association was registered between Haematopus palliatus Temminck, $1820, C$. collaris, $C$. wilsonia, and $H$. himantopus. These, in turn, associated inversely to A. interpres, A. macularia, C. pusilla and C. minutilla (Vieillot, 1819), which is possibly due to the fact that some species are resident, reproducing in the locality, with movements distinct from that specie wich migrate during the boreal winter. Traps, surveys, re-traps, and recovers indicate that the Diamante Branco saline comprises na important area for the conservation of the shorebids.

KEY WORDS. Monitoring, shorebirds, saline, reprodution, conservation
\end{abstract}

O Brasil é visitado periodicamente por milhares de aves limícolas migratórias que se deslocam com a proximidade do inverno boreal, do Ártico para a América do Sul, ocupando várias localidades dentre as quais, o litoral do Nordeste. As espécies que migram para o Nordeste ocupam sobretudo, áreas úmidas naturais no litoral, a exemplo de praias, estuários, manguezais, alagados costeiros e salgados (ANTAS 1986; MORRISON \& ROSS 1989; ANTAS \& NASCIMENTO 1990; RODRIGUES \& ROTH 1990; AZEVEDO \& LARRAZÁBAL 1994).

1) Departamento de Zoologia, Centro de Ciências Biológicas, Universidade Federal de Pernambuco. Avenida Professor Morais Rego 1235, Cidade Universitária, 50670-420 Recife, Pernambuco, Brasil. E-mail: mells@npd.ufpe.br, smaj@npd.ufpe.br

2) Departamento de Biologia, Universidade Federal Rural de Pernambuco. Avenida Dom Manoel de Medeiros, Dois Irmãos, 52171-030 Recife, Pernambuco, Brasil.

3) Salina Diamante Branco, Galinhos, Rio Grande do Norte. 
Entende-se por aves migratórias, aquelas espécies que sazonalmente se deslocam para uma determinada região, utilizando-a como área de invernada ou para a reprodução e por área de invernada, local escolhido pelas aves para alimentação, troca da plumagem, realização das mudas e ganho de peso. A costa brasileira constitui área de invernada para as aves limícolas neárticas. Geralmente essas aves chegam aos locais de invernada no período de agosto a setembro, permanecendo até abril. Indivíduos jovens, subadultos e adultos que não concluiram o ciclo de mudas podem ser observados no litoral de maio a julho. (MORRISON 1984; ANTAS 1984; MIKICH \& LARA 1996; AZEVEDO et al. 2001).

O Parque Nacional da Lagoa do Peixe no Rio Grande do Sul, o Golfo Maranhense e o litoral de Pernambuco, constituem-se em áreas de monitoramento de aves limícolas desde os anos oitenta (ANTAS \& NASCIMENTO 1990; AZEVEDO \& LARRAZÁBAL 1999; RODRIGUES 2000).

Com o objetivo monitorar as espécies de aves limícolas que utilizam ambientes hipersalinos do Rio Grande do Norte foi realizado o presente estudo. Dessa forma pretende-se colaborar com os compromissos assumidos pelo Brasil nas convenções de Washington e de Ransar, que tratam da proteção de aves migratórias e da conservação das aves aquáticas, respectivamente.

\section{MATERIAL E MÉTODOS}

A Salina Diamante Branco está localizada no município de Galinhos, litoral Norte do Rio Grande do Norte (05'05'26"S e 366'31'W), em uma região que constitui-se no mais importante polo salineiro do Brasil. Apresenta uma área com cerca de 5.000 hectares, entre ambientes naturais e antrópicos. Os estudos foram realizados nas áreas naturais: manguezais, praias e estuários dos rios Thomas e Galinhos e em áreas antrópicas (tanques de evaporação e cristalizadores). Para o levantamento qualitativo, foram também consideradas as áreas naturais de dunas e salgados no domínio da Salina Diamante Branco.

As aves foram de acordo com PRATER et al. (1977), HAYMAN et al. (1986) e SICK (1997). Foi registrado o período de reprodução de algumas espécies.

Os censos foram realizados em quatro expedições, sendo a I - 30 de novembro a 08 de dezembro de 1998; II - 12 a 19 de março de 1999 ; III - 10 a 17 de agosto de 1999; IV - 19 a 25 de novembro de 1999. Foi realizada a contagem direta dos indivíduos com o auxílio de um binóculo e uma luneta. Os indivíduos foram contados num percurso diário de $15 \mathrm{Km}$, entre os tanques de evaporação (MORRISON \& ROSS 1989 e MORRISON et al. 1998). Foram realizados observações sobre a alimentação de alguns indivíduos nos tanques de evaporação, assim como o período de reprodução de algumas espécies que ali nidificam.

A Constância das aves contadas foi verificada através da fórmula $\mathrm{C}=\mathrm{p} \mathrm{x}$ 100/P, onde: p corresponde ao número de contagens contendo a espécie e $\mathrm{P}$ o número total de contagens (DAJOZ 1973). Dessa forma, as espécies foram classificadas em constante (quando presente em mais de $50 \%$ das contagens), acessória (quando presente de 25 a $50 \%$ das contagens) e acidental (quando presente em menos de $25 \%$ das contagens). 
Foi estimada a média aritmétrica dos indivíduos contados nas quatro expedições.

A Diversidade Específica foi calculada pelo índice de SHANNON (1948) através da fórmula $\mathrm{H}^{\prime}=-\Sigma \mathrm{pi} \log 2 \mathrm{pi}$, onde $\mathrm{pi}=\mathrm{ni} / \mathrm{N} ; \mathrm{ni}=$ número de indivíduos da espécie i e $\mathrm{N}=$ número total de indivíduos e, a Eqüitabilidade (E) pelo índice de PIELOU (1977), através da fórmula J' = H'/1n S, onde S = número de espécies. Nesse cálculo foi utilizado p programa computacional Ecologia (Measures of Community and Measures of Community Similarity), segundo BROWNER \& ZAR (1984).

A Associação das Amostras foi realizada através da correlação momentoproduto de Pearson, após logaritmização da matriz dos dados de espécies $(\log \mathrm{x}+$ 1), tendo-se utilizado o método de ligação do peso proporcional - WPGMA (Weight Pair Group Mathematical Arithmetic), utilizando-se o programa computacional NTSYS (Numerical Taxonomy and Multivariate Analisys System) da Metagraphics Software Corporation, California, USA, segundo ROHLF (1994).

A Análise dos Componentes Principais (ACP) foi feita com base na correlação momento-produto de Pearson após logaritmização da matriz de dados das espécies $(\log x+1)$ e extraiu-se o auto valor e o auto vetor (LEGENDRE \& LEGENDRE 1998).

\section{RESULTADOS}

A partir das observações de campo realizadas na Salina Diamante Branco foi possível elaborar a listagem específica das aves limícolas, tendo sido registradas 21 espécies: Haematopus palliatus Temminck, 1820; Vanellus chilensis (Gmelin, 1789); Pluvialis squatarola (Linnaeus, 1758); Charadrius semipalmatus Bonaparte 1825; C. collaris Vieillot, 1818; C. wilsonia Ord, 1814; Arenaria interpres (Linnaeus, 1758); Tringa solitaria Wilson, 1813; T. flavipes (Gmelin, 1789); T. melanoleuca (Gmelin, 1789); Actitis macularia (Linnaeus, 1766); Catoptrophorus semipalmatus (Gmelin, 1780); Calidris canutus (Wilson, 1813); C. minutilla (Vieillot, 1819); C. fuscicollis (Vieillot, 1819); C. pusilla (Linnaeus, 1766); C. alba (Pallas, 1764); C. himantopus (Bonaparte, 1826); Numenius phaeopus Linnaeus, 1758; Limnodromus griseus Gmelin, 1789 e Himantopus himantopus (Müller, 1776). A observação de C. himantopus constitui-se no primeiro registro da espécie para o Estado do Rio Grande do Norte. Foram registrados: um filhote de Charadrius collaris em 11 de dezembro de 1998, ao lado dos pais correndo sobre o paredão de um evaporador; um ninho contendo três ovos de $C$. wilsonia construído na área de lavagem do sal, em 18 de junho de 2000; ninhos com dois ovos de Himantopus himantopus, nas terras altas dos evaporadores em junho e julho de 1999 e 2000. Os censos encontram-se relacionados na tabela I. Arenaria interpres, Tringa flavipes, T. melanoleuca e Calidris pusilla alimentam-se de Artemia franciscana (Kellog, 1996) (Crustacea, Branchiopoda) nos primeiros horários da manhã, tendo sido observado durante os censos diários nos tanques de evaporação da Salina Diamante Branco.

A tabela II demonstra a constância observada entre as espécies de aves limícolas de acordo com as expedições realizadas, tendo sido consideradas constantes: Pluvialis squatarola, Charadrius semipalmatus, Arenaria interpres, Tringa flavipes, T. melanoleuca e Actitis macularia. 
Tabela I. Média das contagens de aves limícolas em quatro expedições na Salina Diamante Branco, Galinhos, Rio Grande do Norte, onde a expedição I foi realizada de 30 de novembro a 8 de dezembro de 1998, a II de 12 a 19 de março de 1999, a III de 10 a 17 de agosto de 1999 e a IV de 19 a 25 de novembro de 1999.

\begin{tabular}{lcccc}
\hline \multicolumn{1}{c}{ Espécie } & Expediçãol & Expedição II & Expedição III & Expedição IV \\
\hline Haematopus palliatus & - & 3 & 2 & 2 \\
Vanellus chilensis & - & - & 2 & 5 \\
Pluvialis squatarola & 18 & 12 & 16 & 18 \\
Charadrius semipalmatus & 26 & 24 & 37 & 89 \\
Charadrius collaris & 2 & 4 & 11 & 2 \\
Charadrius wilsonia & - & 4 & 4 & 2 \\
Arenaria interpres & 464 & 302 & - & 179 \\
Tringa solitaria & 1 & 1 & 103 & 266 \\
Tringa flavipes & 171 & 407 & 6 & 227 \\
Tringa melanoleuca & 232 & 315 & - & 21 \\
Acititis macularia & 20 & 16 & - & 3 \\
Catoptrophorus semipalmatus & 1 & - & 2 & 18 \\
Calidris canutus & - & 13 & 0 & 1029 \\
Calidris minutilla & 17 & 8 & 70 & 8 \\
Calidris fuscicollis & 172 & 3 & 9 & - \\
Calidris pusilla & 824 & 1455 & - & 20 \\
Calidris alba & 25 & 10 & 20 & - \\
Calidris himantopus & - & 19 & 193 & 5 \\
Numenius phaeopus & 21 & 10 & 692 & 2204 \\
Limnodromus griseus & 29 & 52 & & \\
Himantopus himantopus & - & 2675 & & \\
\hline Total & 2023 & & & \\
\hline
\end{tabular}

Tabela II. Constância das espécies de aves limícolas evidenciadas a partir de censos na Salina Diamante Branco, em: 30/11 a 08/12/1998 (1), 12/03 a 19/03/1999 (2), 10/08 a 17/08/1999 (3) e 19/11 a 25/11/1999 (4).

\begin{tabular}{|c|c|c|c|}
\hline Espécie & Constantes & Acessórias & Acidentais \\
\hline Haematopus palliatus & 3 & 2,4 & \\
\hline Vanellus chilensis & & 3 & 4 \\
\hline Pluvialis squatarola & $1,2,34$ & & \\
\hline Charadrius semipalmatus & $1,2,3,4$ & & \\
\hline Charadrius collaris & $2,3,4$ & & 1 \\
\hline Charadrius wilsonia & 3,4 & 2 & \\
\hline Arenaria interpres & $1,2,3,4$ & & \\
\hline Tringa solitaria & & 2 & 1,4 \\
\hline Tringa flavipes & $1,2,3,4$ & & \\
\hline Tringa melanoleuca & $1,2,3,4$ & & \\
\hline Actitis macularia & $1,2,3,4$ & & \\
\hline Catoptrophorus semipalmatus & & & 1 \\
\hline Calidris canutus & & 2,4 & \\
\hline Calidris minutilla & $1,2,4$ & 3 & \\
\hline Calidris fuscicollis & 1 & 2,4 & \\
\hline Calidris pusilla & $1,2,3,4$ & & \\
\hline Calidris alba & $1,2,3,4$ & & \\
\hline Calidris himantopus & & 2 & \\
\hline Numenius phaeopus & $1,2,3,4$ & & \\
\hline Limnodromus griseus & 1,2 & & 3 \\
\hline Himantopus himantopus & 2,3 & & 4 \\
\hline
\end{tabular}


As amostras coletadas na Salina Diamante Branco apresentaram Diversidade Específica média, com valores entre 2,0-3,0 bits.ind ${ }^{-1}$ (Fig. 1), sendo a expedição III aquela de maior diversidade $\left(3,0\right.$ bits.ind $\left.^{-1}\right)$.

Calculando-se a Eqüitabilidade das espécies os valores apresentados foram superiores a 0,5 (Fig. 2), sendo a expedição III de maior equilíbrio com 0,74, demonstrando que as espécies estiveram bem distribuídas nas amostras.
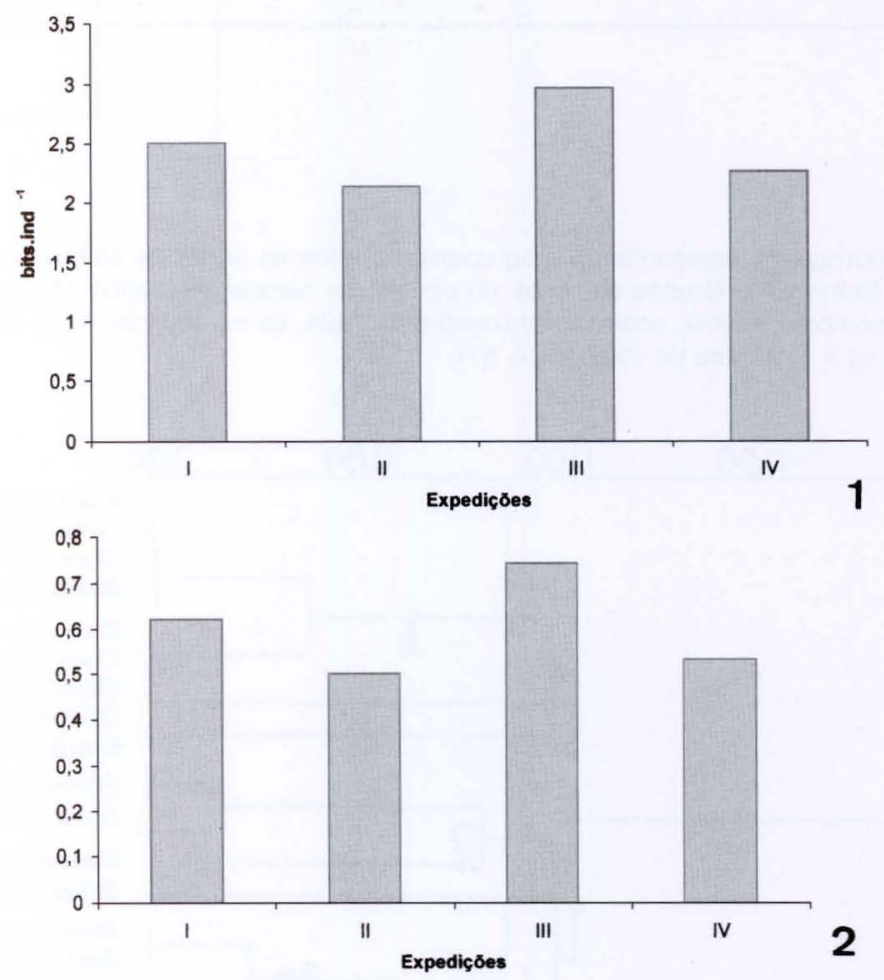

Figs 1-2. (1) Diversidade Específica (DE) e (2) Eqüitabilidade (E) calculadas a partir do índice de diversidade de Shannon (base 2), das amostras coletadas na Salina Diamante Branco, Rio Grande do Norte.

A Análise Cofenética das amostras mostrou $\mathrm{r}=0,57$, estando o dendrograma bem ajustado. A figura 3 explica o agrupamento existente entre os períodos de coleta, nos quais associaram-se os meses de novembro, dezembro e março em um grupo e o mês de agosto ficando separado, coincindo os períodos migratórios das aves limícolas que chegam do hemisfério Norte no início de setembro, permanecendo até abril.

A Análise Cofenética das espécies resultou em $\mathrm{r}=0,89$, estando o dendrograma bem ajustado. A figura 4 demonstra o dendrograma do agrupamento entre as espécies de aves limícolas, onde foram verificados três grupos, sendo o grupo 


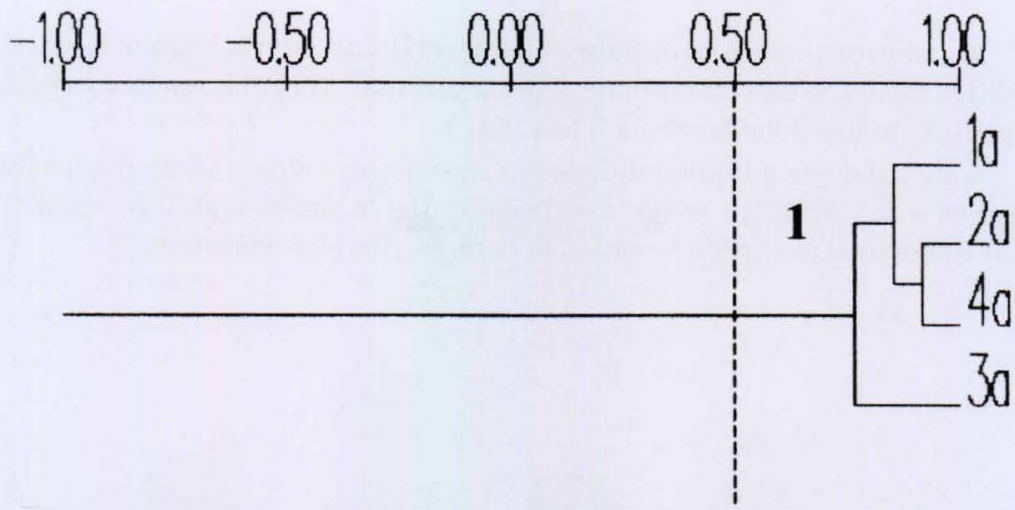

Fig. 3. Dendrograma apresentando o agrupamento entre as amostras coletadas na Salina Diamante Branco, Rio Grande do Norte, no período de censos realizados de dezembro de 1998 a novembro de 1999 , onde $1 \mathrm{a}=$ dezembro de $1998,2 \mathrm{a}=$ março de $1999,3 \mathrm{a}=$ agosto de 1999 e $4 \mathrm{a}=$ novembro de $1999(r=0,57)$.

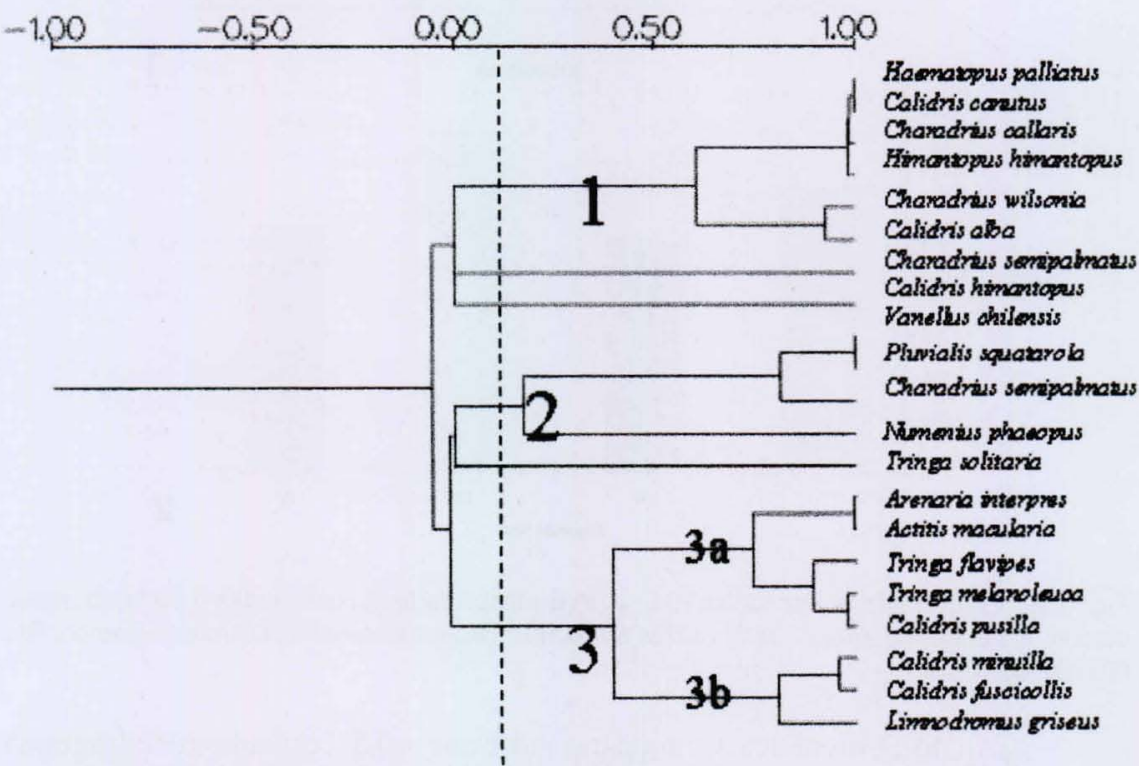

Fig. 4. Dendrograma apresentando os agrupamentos entre as aves limícolas observadas nos censos realizados na Salina Diamante Branco, Rio Grande do Norte, no período compreendido entre dezembro de 1998 a novembro de $1999(r=0.89)$.

1 representado pelas espécies que utilizam as áreas dos paredões de pedras que margeiam os tanques de evaporação e cristalizadores, numa interfácie com o ambiente natural de praias do rio Thomás; nesse grupo foram registrados Hae- 
matopus palliatus, Calidris canutus, C. collaris, Himantopus himantopus, Charadrius wilsonia e Calidris alba; o grupo 2 representado por espécies com preferência por ambientes de manguezais e alagados, constituídos por Pluvialis squatarola, Charadrius semipalmatus e Numenius phaeopus. No grupo 3 foram constatados dois subgrupos, sendo o primeiro deles (3a) representado por espécies com preferências pelo ambiente dos tanques de evaporação, a exemplo de Arenaria interpres, Actitis macularia, Tring a flavipes, T. melanoleuca e Calidris pusilla. O segundo subgrupo (3b) apresentou espécies com preferência pelo ambiente de manguezal, a exemplo de Calidris minutilla, C. fuscicollis e Limnodromus griseus.

$\mathrm{Na}$ tabela III da Análise dos Componentes Principais (ACP), os dois primeiros fatores explicaram 93,85\%, da variação dos dados. Fator 1 - Associou diretamente entre si Haematopus palliatus, Charadrius collaris, C. wilsonia e Himantopus himantopus, e todos estes, associados inversamente a Vanellus chilensis, Arenaria interpres, Actitis macularia, Calidris minutilla e C. pusilla. Fator 2, associou diretamente entre si Tringa flavipes, T. melanoleuca e Calidris canutus, estando inversamente associados a Pluvialis squatarola e Numenius phaeopus.

Tabela III. Análise dos componentes principais das Aves limícolas na Salina Diamante Branco, Galinhos, Rio Grande do Norte, demonstrando os valores pela Correlação Momento-Produto de Paerson entre os três primeiros fatores e os respectivos taxa. Os valores de correlação maiores do que 0,6 estão demonstrados em negrito.

\begin{tabular}{|c|c|c|c|}
\hline Taxa & Fator $1(55,92 \%)$ & Fator $2(37,93 \%)$ & Fator $3(24,53 \%)$ \\
\hline \multicolumn{4}{|l|}{ Haematopodidae } \\
\hline Haematopus palliatus & 1,000 & 0,150 & 0,343 \\
\hline \multicolumn{4}{|l|}{ Charadriidae } \\
\hline Vanellus chilensis & $-1,000$ & 0,243 & 0,501 \\
\hline Pluvialis squatarola & $-0,484$ & $-0,691$ & $-0,103$ \\
\hline Charadrius semipalmatus & $-0,341$ & $-0,576$ & 0,649 \\
\hline Charadrius collaris & 1,000 & 0,150 & 0,148 \\
\hline Charadrius wilsonia & 0,817 & 0,572 & $-0,386$ \\
\hline \multicolumn{4}{|l|}{ Scolopacidae } \\
\hline Arenaria interpres & $-1,000$ & $-0,031$ & $-0,094$ \\
\hline Tringa solitaria & 0,000 & 0,000 & 0,000 \\
\hline Tringa flavipes & $-0,493$ & 0,812 & 0,460 \\
\hline Tringa melanoleuca & $-0,665$ & 0,812 & 0,179 \\
\hline Actitis macularia & $-0,997$ & 0,033 & $-0,062$ \\
\hline Catoptrophorus semipalmatus & 0,000 & 0,000 & 0,000 \\
\hline Calidris canutus & 0,404 & 1,000 & $-0,260$ \\
\hline Calidris minutilla & $-0,878$ & 0,409 & $-0,512$ \\
\hline Calidris fuscicollis & $-0,531$ & $-0,788$ & $-0,849$ \\
\hline Calidris pusilla & $-0,752$ & 0,749 & 0,175 \\
\hline Calidris alba & $-0,027$ & 0,287 & $-1,000$ \\
\hline Calidris himantopus & 0,000 & 0,000 & 0,000 \\
\hline Numenius phaeopus & 0,164 & $-0,860$ & $-0,409$ \\
\hline Limnodromus griseus & $-0,541$ & 0,510 & $-0,724$ \\
\hline \multicolumn{4}{|l|}{ Recurvirostridae } \\
\hline Himantopus himantopus & 0,988 & 0,313 & $-0,283$ \\
\hline Soma dos três fatores & & $118,37 \%$ & \\
\hline
\end{tabular}




\section{DISCUSSÃO}

Calidris pusilla, Arenaria interpres, Tringa flavipes e T. melanoleuca foram as aves mais numerosas nas contagens, sobretudo em março, período este, próximo ao retorno das populações para as áreas reprodutivas no Ártico (MORRISON 1984; HAYMAM et al. 1986). Trabalhos realizados por NASCIMENTO \& LARRAZÁBAL (2000) no litoral do Rio Grande do Norte indicaram Charadrius semipalmatus e Calidris fuscicollis como espécies mais abundantes, divergindo dos resultados aqui obtidos.

A constância observada em Pluvialis squatarola, Charadrius semipalmatus, Arenaria interpres, Tringa flavipes, T. melanoleuca, Actitis macularia, Calidris pusilla, C. alba e Numenius phaeopus no litoral norte do Rio Grande do Norte difere daqueles encontrados por AZEVEDO \& LARRAZÁBAL $(1994,1999)$ para outras localidades do Nordeste.

A associação positiva entre Haematopus palliatus, Charadrius collaris, $C$. wilsonia e Himantopus himantopus e, todos estes, associados inversamente a Arenaria interpres, Actitis macularia, Calidris pusilla e C. minutilla deve-se ao fato de algumas espécies serem residentes, realizando a reprodução na localidade, com movimentação distinta daquelas neárticas, que se movimentam com a proximidade do inverno boreal (SICK 1983; MORRISON 1984; MORRISON \& ROSS 1989).

A adaptabilidade de Artemia franciscana quanto à temperatura, salinidade e oxigênio em ambientes hipersalinos (CÂMARA \& CASTRO 1983) favorece a sua multiplicação constituindo-se em um recurso disponível para alimentação de aves limícolas, a exemplo de Arenaria interpres, Tringa flavipes, T. melanoleuca e Calidris pusilla nos tanques de evaporação da Salina Diamante Branco. NASCIMENTO \& LARRAZÁBAL (2000) estudando a alimentação das aves em tanques de cultivo de camarão, instalados em antigas salinas em Barra de Cunhaú, litoral Sul do Rio Grande do Norte, não mencionam esse microcrustáceo como item alimentar, relacionando anelídeos poliquetos e outros crustáceos, sendo este, um dos poucos trabalhos sobre alimentação de limícolas em áreas antrópicas do litoral do Nordeste.

As capturas, recapturas e recuperações, como também, os resultados dos censos, indicam que a região da Salina Diamante Branco no município de Galinhos, constitui uma importante área de invernada, sobretudo para Arenaria interpres, Tringa flavipes, T. melanoleuca e Calidris pusilla; é local de reprodução para Charadrius collaris, C. wilsonia e Himantopus himantopus (SICK 1997).

AGRADECIMENTOS. Á Salina Diamante Branco, Universidade Federal Rural de Pernambuco, Universidade Federal de Pernambuco e ao Centro de Pesquisa para Conservação das Aves Silvestres pelo apoio ao presente trabalho. Agradecemos também ao Prof. Dr. Antônio Rossano Mendes Pontes e à Profa. Dra. Sigrid Neumann-Leitão pelas contribuições ao texto.

\section{REFERÊNCIAS BIBLIOGRÁFICAS}

ANTAS, P.T.Z. 1984. Migration of neartic shorebids (Charadriidae and Scolopacidae) in Brazil -flywas and their different seasonal use. Wader Study Group Bull., Tring, 39: 52-56.

1986. Migração de aves no Brasil, p. 153-187. In: Anais do Encontro Nacional de Anilhadores de Aves, 2, Rio de Janeiro, 1986. Rio de Janeiro, Univ. Fed. do Rio de Janeiro, 187p.

Revta bras. Zool. 19 (4): 1081 - 1089, 2002 
Antas, P.T.Z. \& I.L.S Nascimento. 1990. Análise dos dados de anilhamento de Calidris pusilla no Brasil, p. 6-12. In: Anais do Encontro Nacional de Anilhadores de Aves, 4, Recife, 1988. Recife, Univ. Fed. Rural de Pernambuco, 151p.

Azevedo Jr., S.M. DE \& M.E. Larrazábal. 1994. Censo de aves limícolas na Coroa do Avião, Pernambuco, Brasil, informações de 1991 a 1992. Rev. Nord. Zool., Recife, 1 (1): 263-277.

- 1999. Captura e anilhamento de Calidris pusilla (Scolopacidae) na costa de Pernambuco. Ararajuba 7 (2): 63-69.

Azevedo JR., S.M. DE; M.M. Dias; M.E. LarrazÁbal; W.R. Telino JR; R.M. Lyra-Neves \& C.J.G. FERNANDES. 2001. Recapturas e recuperações de aves migratórias no litoral de Pernambuco, Brasil. Ararajuba 9 (1): 33-42.

BRownER, J. \& J.H. ZAR. 1984. Field and Laboratory methods for general ecology. Dubuque, Iowa, $2^{\text {nd }}$ ed., 226p.

CÂMARA, M.R. \& E.V. CASTRo. 1983. Artemia salina L. (Anacostraca): uma opção para a aqüicultura do Nordeste do Brasil. Revta bras. Zool. 1 (3): 145-147.

DAJoz, R. 1973. Ecologia Geral. Petrópolis, Vozes, 472p.

HAYMAn, P.; J. Marchant \& T. PRATER. 1986. Shorebirds: an identification guide. Boston, Houghton Mifflin Co., 412p.

LegendRe, P. \& L. Legendre. 1998. Numerical ecology. Amsterdam, Elsevier, 853p.

Mikich, S. B. \& A.I. LARA. 1996. Levantamento das aves limícolas da praia Deserta, ilha de Superagui, Guaraqueçaba (Paraná-Brasil). Estudos de Biologia, Curitiba, 4 (40): 55-70.

MorRISON, R.G.I. 1984. Migration systems of some new world shorebirds, p. 125-202. In: J. BURGER \& B.L. OlLA (Eds). Behavior of marine animals. Shorebirds: migration and forangins behavior. New York, Plenum Press, vol. 6, 743p.

Morrison, R.G.I. \& R.K Ross. 1989. Atlas of Neartic shorebirds on the coast of South America. Ottawa, Canadian Wildlife Service, Vol. 1, 128p.

Morrison, R.I.G.; R.W. Butler; F.S. Delgado \& R.K. Ross. 1998. Atlas of Neartic shorebirds and other waterbirds on the coast of Panamá. Ottawa, Canadian Wildlife Service, 112p.

NASCIMENTO, J.L.X. Do \& M.E. LARRAZÁBAL. 2000. Alimentação de aves limícolas em Barra de Cunhaú, Canguaretama, Rio Grande do Norte. Melopsittacus 3 (3): 91-109.

Pielou, E.C. 1977. Mathematical Ecology. New York, Wiley, 385p.

Prater, T.; J. Marchant \& J. Vourinen. 1977. Guide to identification and ageing of Holartic Waders. British Trust for Ornithology, Field Guide 17. Maund and Irvine, Herts, 168p.

Rodrigues, A.A.F. 2000. Seasonal abundance of neartic shorebirds in the gulf of Maranhão, Brazil. Jour. Field Ornithol. 71 (4): 665-673.

Rodrigues, A.A.F. \& P.G. RotH. 1990. Distribuição e fenologia de várias espécies de maçaricos e batuíras em parte da costa Oeste da Ilha de São Luís - Maranhão, p. 44-58. In: Anais do Encontro Nacional de Anilhadores de Aves, 4. Recife, 151p.

RoHLF, F.J. 1994. NTSYS-PC Numerical taxonomy and multivariate analysis system. New York, Exetor Software, Applied Biostatistics Inc., 133p.

SHAnnon, L.E. 1948. A mathematical theory of communication. Bul. Syst. Tech. Jour. 27: 379-423.

Sick, H. 1983. Migração de Aves na América do Sul continental. Brasília, Inst. Bras. de Desenv. Florestal, Vol. 2, 86p.

- 1997. Ornitologia brasileira. Rio de Janeiro, Ed. Nova Fronteira, 912p.

Recebido em 13.III.2002; aceito em 09.X.2002. 